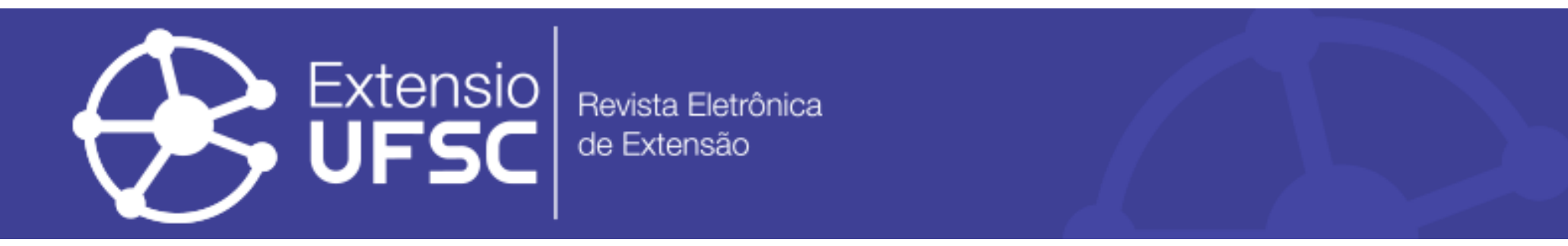

\title{
O ENSINO AO FAMILIAR CUIDADOR DURANTE A TERMINALIDADE: RELATO DE EXPERIÊNCIA
}

\author{
Anderson Roberto de Sales Corrêa \\ Universidade Federal do Pará \\ anderson.sales13@gmail.com \\ Mary Elizabeth de Santana \\ Universidade do Estado do Pará \\ betemary@terra.com.br
}

\author{
Cinthia Costa de Castro \\ Secretaria Estadual de Saúde Pública do Pará \\ cinthia_ccastro@hotmail.com \\ Wagner Luiz Oliveira Ximenes \\ Universidade do Estado do Pará \\ wagner.uepa@gmail.com \\ Ana Cláudia de Matos Santos \\ Universidade do Estado do Pará \\ scpo.unacon@gmail.com
}

\section{Resumo}

Objetivo: Descrever experiências de orientação de paciente e cuidador em um projeto de extensão em cuidados paliativos entre 2013 e 2014. Metodologia: trata-se de um relato de experiência das vivências educativas de um projeto de extensão em cuidados paliativos de um hospital universitário em Belém vinculado a UFPA. Resultados: As orientações foram relacionadas aos cuidados com horário dos medicamentos ( $\mathrm{n}=37)$; Mudança de decúbito $(\mathrm{n}=36)$; Banho de Aspersão $(\mathrm{n}=28)$; Prevenção de Quedas $(\mathrm{n}=33$ ); Uso de hidratante $(n=33)$; Massagem de conforto $(n=18)$ e Sinais de Urgência $(n=19)$. As estratégias de ensino-aprendizagem foram: cartilhas, orientações escritas e verbais, demonstração do cuidado e folder. Conclusão: $O$ estudo possibilitou demonstrar a potencialidade do projeto de extensão para educação em saúde a nível hospitalar para a permanência do sujeito com em cuidado paliativo no seu lar, diminuindo as intercorrências relacionada ao cuidado de enfermagem e aumentando a segurança de cuidadores e paciente no cuidado domiciliar.

Descritores: Cuidados Paliativos. Enfermagem. Educação em Saúde. Pacientes Domiciliares.

\section{TEACHING THE FAMILY CAREGIVER DURING TERMINALITY: EXPERIENCE REPORT}

\begin{abstract}
Objective: To describe experiences of patient and caregiver orientation in an extension project in palliative care between 2013 and 2014. Methodology: this is an experience report of the educational experiences of an extension project in palliative care of a university hospital in Belém linked to UFPA. Results: The guidelines were related to care with medication schedule ( $\mathrm{n}=37$ ); Change of decubitus ( $\mathrm{n}=36$ ); Sprinkler Bath $(\mathrm{n}=28)$; Fall Prevention $(\mathrm{n}=33)$; Use of moisturizer $(\mathrm{n}=33)$; Comfort massage ( $\mathrm{n}$ $=18$ ) and Signs of Urgency $(n=19)$. The teaching-learning strategy were: booklets, written and verbal guidelines, demonstration of care and folder. Conclusion: The study made it possible to demonstrate the potential of the extension project for health education at the hospital level for the subject's stay in palliative care at home, reducing the intercurrences related to nursing care and increasing the safety of caregivers and patients in care domicile.
\end{abstract}

Keywords: Palliative Care. Nursing. Health Education. Homebound Persons.

\section{LA ENSEÑANZA AL FAMILIAR CUIDADOR DURANTE LA TERMINALIDAD: RELATO DE EXPERIENCIA}

\footnotetext{
Resumen

Objetivo: Describir experiencias de orientación de paciente y cuidador en un proyecto de extensión en cuidados paliativos entre 2013 y 2014. Metodología: se trata de un relato de experiencia de las vivencias educativas de un proyecto de extensión en cuidados paliativos de un hospital universitario en Belém vinculado a la UFPA. Resultados: Las orientaciones se refirieron a los cuidados con horario de los medicamentos ( $\mathrm{n}=37$ ); Cambio de decúbito $(\mathrm{n}=36)$; Baño de Aspersión ( $\mathrm{n}=28$ ); Prevención de caídas $(\mathrm{n}=$ 33); Uso de hidratante $(\mathrm{n}=33)$; Masaje de confort $(\mathrm{n}=18)$ y Señales de Urgencia $(\mathrm{n}=19)$. La estrategia de enseñanza-aprendizaje fueron: cartillas, orientaciones escritas y verbales, demostración del cuidado y folder. Conclusión: El estudio posibilitó demostrar la potencialidad del proyecto de extensión para educación en salud a nivel hospitalario para la permanencia del sujeto con en cuidado paliativo en su hogar, disminuyendo las intercurrencias relacionadas al cuidado de enfermería y aumentando la seguridad de cuidadores y paciente en el cuidado casa.

Palabras clave: Cuidados Paliativos. Enfermería. Educación en Salud. Personas Imposibilitadas.
} 
O ensino ao familiar cuidador durante a terminalidade: relato de experiência

\section{INTRODUÇÃO}

Hoje, a ciência médica pode lutar contra uma doença potencialmente fatal e a morte, quando antes apenas podiam ser oferecidos conforto e segurança. Com o avançar das tecnologias em saúde e mudanças na filosofia do ensino em saúde, tornou-se comum o prolongamento da vida a qualquer custo. Nesse contexto, a morte passa a ser entendida como um fracasso e, por esse motivo, deve ser escondida. O hospital raramente está preparado para tratar e cuidar do sofrimento do paciente e família. Ajudar indivíduos com doenças que ameaçam a continuidade da vida e seus familiares em um dos momentos mais cruciais é uma atividade ou modelo de atenção à saúde denominado cuidado paliativo (MACIEL et al, 2006).

O cuidado paliativo preza a finitude digna, aquela sem dor e com sofrimento minimizado a partir dos cuidados adequados. A terapêutica paliativa é voltada ao controle sintomático e preservação da qualidade de vida para o paciente (INCA, 2002). Ao se realizar estudo de necessidade de cuidados paliativos na atenção primária, foi identificado que, em todas as equipes de Estratégia Saúde da Família, havia paciente com necessidade desta modalidade terapêutica. Foi evidenciado longa espera para consultas agendadas e falta de visitas domiciliares. Os autores afirmam que o cuidado paliativo baseado na comunidade tem sido associado a melhor controle dos sintomas, satisfação do paciente, menor custo e menor taxa de admissão hospitalar (MARCUCCI et al, 2016).

Este estudo descreve as ações de educação em saúde, por meio de um projeto de extensão, em um serviço de cuidados paliativos oncológico, realizado em um hospital universitário na cidade de Belém/PA vinculado a Universidade Federal do Pará. Relata a experiência de um discente do curso de graduação em enfermagem inserido no projeto de extensão "Cuide bem do seu paciente" direcionado a pacientes com câncer avançado e em cuidado paliativo que retornarão para o domicílio e seus cuidadores e familiares.

As atividades de educação em saúde ao paciente direcionadas para o cuidar de si, preserva a autonomia do paciente em cuidados paliativos e de seu familiar cuidador. É essencial para a internação em seu domicílio, pois visa a prevenção de agravos à saúde relacionado à internação hospitalar e autonomia para a realização dos cuidados básicos prestados em domicílio. A enfermagem se insere no processo de ensino e aprendizagem 
O ensino ao familiar cuidador durante a terminalidade: relato de experiência

voltado para o indivíduo; preservando a autonomia do paciente e mantendo a inserção em seu meio familiar e sócio cultural. O estudo teve como objetivo descrever a experiência das estratégias educativas na atenção terciária destinada ao paciente e cuidador vivenciando os cuidados paliativos oncológicos em preparo de internação domiciliar.

\section{MATERIAIS E MÉTODOS}

Este trabalho trata-se de um estudo descritivo, do tipo relato de experiência das vivências educativas de um discente do curso de enfermagem que esteve inserido como bolsista no projeto de extensão cujo título é "Cuidados paliativos: cuide bem do seu paciente" desenvolvido no período Março de 2013 a março de 2014, em um hospital universitário.

Como instrumento de coleta de dados para verificar as necessidades de orientações ao cuidado, foi elaborado um formulário para preenchimento das necessidades humanas afetadas e as intervenções de enfermagem que o cuidador pode realizar. $O$ instrumento também foi utilizado para prever novos agravos e orientar os cuidados, para que ele não ocorra, e sobre a organização do serviço. As orientações foram realizadas pelo bolsista de enfermagem, por meio de exposição dialogada dirigida e individualizada, com demonstração prática do cuidado a ser realizado, utilização de instrumentos de ensino como: folders, cartilhas e álbuns seriados. Caso ocorresse alguma necessidade do paciente em que não houvesse material ilustrativo preparado, ou em situações em que o cuidador principal não está presente para receber as orientações, era fornecido ao paciente e acompanhante orientações escritas e detalhadas sobre os cuidados e condutas relacionadas às intervenções de enfermagem.

Teve como local de orientação as clínicas de internação hospitalar ou o ambulatório de cuidados paliativos, com sessões de 20 a 30 minutos dependendo da necessidade de cuidados, para a melhor compreensão dos cuidados, foi solicitada a execução do cuidado e, se necessário, reforço das orientações com novas sessões educativas. As propostas e execução das atividades de educação em saúde foi baseada na nova perspectiva de bancária, definida como uma maneira de ensinar que respeita o aluno 
O ensino ao familiar cuidador durante a terminalidade: relato de experiência

e o estimula, oferecendo condições para que trabalhe ativamente em benefício do seu próprio enriquecimento intelectual, social e afetivo (LINS, 2011).

A avaliação da necessidade de orientações e coleta de dados foi realizada por instrumento específico orientado pelas necessidades humanas básicas afetadas durante o transcorrer e avanço da doença. Este modelo é proposto por Wanda de Aguiar Horta, desenvolvido a partir da teoria de Maslow, e fundamenta as necessidades humanas básicas (NHB) em: Necessidades fisiológicas; segurança; amor; estima e autorrealização (HORTA, 1974). Os resultados obtidos, bem como as intercorrências ocorridas em domicílio relacionadas ao avanço da doença e ao cuidado realizado foi registrado em um formulário de estatística de resultados do projeto.

Para avaliação do processo de educação em saúde, foram utilizadas as intercorrências relacionada ao cuidado domiciliar, registro das notificações das intercorrências do telefone do serviço, e verbalização do cuidador e paciente durante consulta ambulatorial.Este estudo dispensou apreciação ética por se tratar de um relato de experiência dos autores envolvidos na produção do trabalho.

\section{RESULTADOS E ANÁLISES}

O objetivo central deste projeto de extensão foi orientar pacientes com câncer avançado sob cuidados paliativos e seus acompanhantes e demais familiares que retornarão para suas residências, sobre os cuidados que podem e devem ser realizados no domicílio. A execução das atividades iniciou a partir do mês de Março de 2013. Após a seleção, o discente foi capacitado, através de acompanhamento e orientação sobre a organização do serviço e a filosofia da linha de cuidados paliativos. As orientações foram realizadas na enfermaria, ambulatório de cuidados paliativos e no domićlio durante visita domiciliar. Com reforço das orientações em consultas de retorno.

A equipe do Serviço de Cuidados Paliativos em Oncologia (SCPO) é multiprofissional e interdisciplinar, acrescido das contribuições da equipe assistente das clínicas; realiza interconsultas nas enfermarias do hospital e atende em ambulatório, com rotinas de visita domiciliar. As visitas diárias na internação são feitas por equipe multidisciplinar. O hospital dispõe de um Pronto Atendimento em oncologia, onde os 
O ensino ao familiar cuidador durante a terminalidade: relato de experiência

pacientes oncológicos podem dirigir-se caso ocorra alguma intercorrência de urgência relacionada ao câncer ou não, e de telefone móvel 24 horas onde os pacientes e cuidadores podem sanar dúvidas sobre condutas que podem ser feitas no domicílio e para o manejo de intercorrência de urgências

Durante o transcorrer das atividades de extensão foram orientados 50 pacientes e familiares com perfil e condições clínicas de internação domiciliar. Quanto ao sexo, 24 eram do sexo masculino e 26 do sexo feminino; em relação a procedência, 3 foram encaminhados pela clínica de Doenças Infecto-parasitárias, 11 pela clínica cirúrgica, 6 pela clínica de pneumologia, 16 pelo serviço de oncologia clínica, 1 pelo serviço de radioterapia, 14 pela clínica médica e o restante por demanda espontânea ou desistência do tratamento ativo curativo.

Em relação a demanda de pacientes, foram encaminhados novos pacientes para o serviço: 15 no primeiro trimestre, 13 no segundo trimestre, 9 no terceiro trimestre e 13 no quarto trimestre.. Os locais para as orientações de enfermagem foram a enfermaria $(\mathrm{n}=29)$, ambulatório $(\mathrm{n}=12)$, domicílio $(\mathrm{n}=2)$.

Anteriormente a execução das atividades de educação em saúde, era realizada investigação do conhecimento preexistente dos cuidadores e pacientes; e capacidade para compreensão das orientações de enfermagem e segurança para executá-las no domicílio.

As necessidades de orientações de cuidado eram coletadas a partir da avaliação de enfermagem, considerando as necessidades humanas básicas afetadas ou dispositivos médico instalado durante a internação hospitalar, durante o processo de terminalidade eram coletados a partir de impresso específico e individualizado por binômio famíliapaciente e posteriormente programada as intervenções educativas (Tabela 1).

Tabela 1 - Modelo de impresso de necessidades de orientação de cuidado

\begin{tabular}{|l|l|l|}
\hline $\begin{array}{l}\text { Problemas/NHB } \\
\text { afetadas }\end{array}$ & Intervenções proposta & $\begin{array}{l}\text { Data programada da } \\
\text { atividade educativa }\end{array}$ \\
\hline & & \\
\hline & & \\
\hline & & \\
\hline
\end{tabular}


O ensino ao familiar cuidador durante a terminalidade: relato de experiência

As necessidades de cuidado foram relacionadas a: mobilidade física prejudicada $(n=19)$, banho de aspersão ou banho no leito $(n=18)$, dor $(n=15)$, emagrecimento ou caquexia $(n=14)$, desconforto respiratório $(n=13)$, edema $(n=10)$, fragilidade cutânea $(n=8)$, inapetência $(n=7)$, pele desidratada e lesão por pressão $(n=5)$ cada; enfrentamento ineficaz, fadiga, constipação e ferida tumoral $(n=3)$ cada, ascite ou distensão abdominal, gastrostomia ou jejunostomia $(n=4)$ cada, diarreia, náuseas, cateter vesical $(n=1)$ cada, as necessidades de cuidado foram identificadas a partir dos sintomas e sinais apresentados pelo paciente e dúvidas do cuidador e familiar.

Em estudo observacional, descritivo, quantitativo, retrospectivo desenvolvido por Sousa (2012) em uma unidade de cuidados paliativos oncológicos em Portugal, foi identificado como principais sintomas no momento da admissão hospitalar: dor, dispneia, xerostomia, sede, anorexia, disfagia, náuseas, vômitos, constipação, ansiedade, insônia, confusão, tristeza e astenia. Esses dados apresentados são compatíveis com o exposto no presente estudoAs orientações de enfermagem foram relacionadas a administração e preparo de medicamentos por gastrostomia e jejunostomia $(n=6)$; alimentação fracionada e fornecer de acordo com a preferência do paciente $(n=16)$; técnica de realização de curativo de ferida $(n=12)$; horário das medicações utilizando tabela ilustrativa $(n=37)$; manutenção de membros inferiores elevados para diminuição do edema ( $n=16)$; mudança de decúbito $(n=36)$; massagem de conforto $(n=18)$; técnica do Banho de Aspersão ( $n=28)$; dieta Laxativa ( $n=9)$; aumento da ingestão de líquidos $(n=12)$; sinais de encaminhamento ao pronto atendimento em oncologia ou ao hospital de referência do município no interior $(n=19)$; curativo de jejunostomia ou gastrostomia $(n=8)$; administração de dieta e medicamentos por gastrostomia e jejunostomia $(n=5)$; prevenção de quedas ( $n=33)$; uso de hidratante na pele $(n=33)$; técnica do banho no leito ou de aspersão ( $n=16)$; mobilização para alívio do desconforto respiratório $(n=16)$; uso de coxins ou acolchoado $(n=12)$; organização e funcionamento do SCPO $(n=17)$, controle analgésico da dor para ajuste de opioides $(n=4)$; técnicas de transferência da cama $(n=16)$; uso de coxins e acolchoados para alívio de pressão $(n=2)$; efeitos imediatos e tardios da radioterapia ( $\mathrm{n}=1)$; uso de ácido graxo essencial na pele $(\mathrm{n}=9)$.

Em estudo realizado em Pelotas (Rio Grande do Sul), onde foram realizadas ações extensionistas para a promoção da espiritualidade com 52 cuidadores familiar de pacientes 
O ensino ao familiar cuidador durante a terminalidade: relato de experiência

internados no domicílio. As autoras identificam que, antes do falecimento os cuidadores manifestavam necessidade de maior compreensão do processo de morte e morrer durante a terminalidade, onde a espiritualidade foi estratégia essencial para o aperfeiçoamento do processo de aceitação. Sendo a morte identificada como momento de tristeza em dualidade com a resignação do alívio do sofrimento para o paciente enfermo, sendo esta a justificativa mais observada para aceitação da morte (OLIVEIRA et al, 2017).

Quanto as intervenções de enfermagem mais prevalente, Sousa (2012) identificou a administração de fármaco para o controle dos sintomas, oxigenoterapia, nebulização com soro fisiológico, espessante alimentar, fornecimento de apoio emocional, umedecer a mucosa oral e cuidados com a higiene oral. É possível perceber um diferente perfil de intervenções de cuidado entre o estudo em Portugal e o presente estudo, podendo-se inferir uma diferente abordagem frente ao sintoma apresentado pelo paciente da exposta por este estudo.

As intercorrências relacionadas ao cuidado foram escape ou curativo inadequado de gastrostomia e jejunostomia $(n=3), 6$ episódios de queda, cinco de lesão por pressão ou cuidado inadequado de feridas e 3 obstruções de gastrostomia ou jejunostomia.

É de responsabilidade da equipe de enfermagem a educação dos pacientes e suas famílias sobre o cuidado em saúde durante a terminalidade (POTTER; PERRY, 2013). Em revisão sistemática de literatura foi possível observar dados que corroboram com as estratégias e objetivos do projeto de extensão, evidenciando que o autocuidado é um processo e envolve autocapacitação, automonitoramento, educação e interação nos pacientes com câncer (HASANPOUR-DEHKORDI, 2016).

Sendo a educação essencial na promoção do cuidar de si, treinamentos em face-aface e em grupo contribuem para a autogestão e o autotratamento. A alfabetização em saúde e as informações levam ao autocuidado eficiente. Os achados de alguns estudos indicam que as estratégias educacionais podem ser independentes da idade e do status socioeconômico para que qualquer indivíduo em qualquer idade e status socioeconômico seja capaz de obter informações necessárias sobre problemas de saúde. Vários fatores, inclusive socioculturais, desempenham um papel na educação, no cliente e na família. Outro atributo neste estudo foi a interação, descrito como base do autocuidado e 
O ensino ao familiar cuidador durante a terminalidade: relato de experiência

pode ocorrer entre pacientes e familiares, amigos e enfermeiros (HASANPOURDEHKORDI, 2016).

Durante o desenvolvimento das atividades educativas, foi percebido fatores que influenciam fortemente no processo de ensino-aprendizagem. Esses são relacionados ao fator financeiro, relativo aos custos de integrar novamente o paciente no domicílio, o medo da morte iminente do paciente em fase final de vida no domićlio, os sintomas refratários ao tratamento medicamentoso e não medicamentoso, e insegurança da execução dos cuidados sem uma equipe técnica supervisionando. Ao ser identificado esses fatores que influenciam no processo educativo, era dialogado com a equipe multiprofissional para a resolução desses problemas, e posteriormente reiniciado as atividades educativas, assim como o reforço das orientações durante as consultas ambulatoriais e investigação das dúvidas relativas ao cuidado no domicílio.

Nos cuidados paliativos o enfermeiro desenvolve ações de diferentes abrangências quando o paciente se encontra internado ou em domicílio. Neste ambiente ele traça o plano de cuidados, orienta o uso das medicações, de acordo com a prescrição médica, faz e orienta a realização de curativos, coloca sondas e cateteres - orientando sobre o seu manuseio diário - entre outros (UNIC, 2009).

Apesar das diversas limitações e incapacidades que o paciente apresenta nessa fase da doença, o enfermeiro, por meio da demonstração e da educação, procura estimular e promover as adaptações necessárias ao autocuidado, em respeito à preservação da autonomia e da dignidade humana, favorecendo o desenvolvimento das suas habilidades que possam contribuir para o cuidar de si. Para tal, ele orienta o paciente e o cuidador, na prática das ações, apoiando-os física e psicologicamente, sempre que necessário (UNIC, 2009).

Em determinadas situações, promover as condições e estimular o autocuidado pode ser um processo árduo e negado pelo próprio paciente e seus familiares, porque as sequelas da doença ou das terapêuticas instituídas previamente podem provocar alterações na autoconfiança, na autoimagem e na autoestima do doente, que perde a vontade de lutar. Muitas vezes, o paciente tem que ser estimulado e educado a cuidar de uma colostomia, ou de uma traqueostomia, situações desconhecidas e adversas à sua condição prévia de saúde e bem-estar (UNIC, 2009). 
O ensino ao familiar cuidador durante a terminalidade: relato de experiência

Em estudo randomizado realizado sobre a percepção da educação em saúde em cuidados paliativos para cuidadores e pacientes com câncer avançado, houve a identificação que: o termo "cuidados paliativos" apresenta estigma negativo associado a morte, sendo adquirido esta concepção a partir das interações com profissionais de saúde (ZIMMERMMAN et al. 2016).

Este estudo possibilitou mostrar à equipe de enfermagem a potencialidade da educação em saúde precoce para a permanência do sujeito com prognóstico reservado no seu lar, diminuindo as intercorrências relacionada ao cuidado de enfermagem e aumentando a segurança de cuidadores e paciente no cuidado domiciliar. Presume-se que a partir dos dados de experiências educativas, estratégias de ensino-aprendizagem e de avaliação, este estudo irá corroborar para incremento nas atividades educativas de outras unidades de cuidados paliativos, assim como embasamento teórico-científico na avaliação das diversas metodologias de educação em saúde para o cuidar de si.

\section{CONCLUSÃO}

O processo de ensino-aprendizagem direcionado para o cuidado paliativo, a partir do projeto de extensão foi pioneiro no hospital universitário vinculado a UFPa. A educação em saúde de pacientes, cuidadores e familiares proporcionou grande benefício no sentido de fornecer maior segurança aos familiares e cuidadores na casa, assim como a identificação precoce dos sinais de urgência e emergência, direcionando condutas precoces de remoção para o Pronto Atendimento e diminuindo o risco de complicações por atendimento tardio.

O uso da cartilha, folder, exposição dialogada, execução dos cuidados e álbum seriado, que foram utilizados em conjunto ou de maneira individual, foi um facilitador das atividades educativas com melhor compreensão pelos pacientes e cuidadores.

Como fatores que reduzem a eficácia do processo educativo e da permanência na internação domiciliar estiveram presentes a preocupação em relação ao prognóstico do paciente, nível baixo de escolaridade, os sintomas refratários ao tratamento, o medo de realizar os cuidados no domicílio e o medo da morte no domić́lio. 
O ensino ao familiar cuidador durante a terminalidade: relato de experiência

Estratégias educativas que promovam melhor concepção dos cuidados paliativos e sobre a situação de terminalidade na sociedade em geral devem ser empregadas no intuito de auxiliar na melhor compreensão e prestação dos cuidados em paciente que se na modalidade terapêutica paliativa. Fatores sociais e econômicos mostraram grande interferência na concentração durante a prática de educação em saúde. Evidenciando a necessidade de atendimento multiprofissional aos familiar cuidador e pacientes em cuidados paliativos oncológicos.

O projeto de extensão apresentou como fragilidade o encaminhamento tardio dos pacientes para a linha de cuidados paliativo exclusivo ou concomitante, tendo como consequência baixo número de sessões de atividades educativas. Estratégias de reunião de cuidadores em grupos mensais para o compartilhamento das experiências de cuidado no domicílio são propostas a serem implementadas, pois pode aumentar o potencial educativo a partir da educação pelos pares.

\section{REFERÊNCIAS}

HASANPOUR-DEHKORDI, Ali. Self-care Concept Analysis in Cancer Patients: An Evolutionary Concept Analysis. Indian Journal of Palliative Care. v.22, n.4. p.388-94. 2016 Disponível em: <http://www.jpalliativecare.com/article.asp?issn=09731075; year $=2016$; volume $=22 ;$ issue $=4$; page $=388$; epage $=394$; aulast $=$ Hasanpour-Dehkordi >. Acesso em 17 Ago 2017.

HORTA, Wanda de Aguiar. Enfermagem: teoria, conceitos, princípios e processos. Rev Esc Enferm USP. v.8, n.1, p.7-15, 1974. Disponível em: <http://www.scielo.br/pdf/reeusp/v8n1/0080-6234-reeusp-8-1-007.pdf>. Acesso em 25 Nov 2018.

INCA, Insituto Nacional de Câncer. Cuidados paliativos oncológicos: controle de sintomas. Rev. Bras. Cancerol. v. 48, n.1, p.191-211. 2002. Disponível em: < http://www.inca.gov.br/rbc/n_48/v02/pdf/condutas3.pdf.>. Acesso em 16 Ago 2017

LINS, Maria Judith Sucupira da Costa. Educação bancária: uma questão filosófica de aprendizagem. Rev Educ Cult Cotemp. v.8, n.6, p.1-12, 2011. Disponível em: $<$ http://periodicos.estacio.br/index.php/reeduc/article/viewArticle/168>. Acesso em 25 Nov 2018.

MACIEL, Maria Gorette Sales.; RODRIGUES, Luis Fernando.; NAYLOR, Claudia.; BETTEGA, Roberto.; BARBOSA, Sílvia Maria.; BURLÁ, Cláudia.; MELO, Inês Tavares Vale. Critérios de qualidade para os cuidados paliativos no Brasil (ANCP). Rio de 
O ensino ao familiar cuidador durante a terminalidade: relato de experiência

Janeiro: Diagraphic; 2006. Disponível em: <http://paliativo.org.br/criterios-dequalidade-para-os-cuidados-paliativos-no-brasil/>. Acesso em 16 ago 2017.

MARCUCCI, Fernando C.I.; CABRERA, Marcos A.S.; PERILLA, Anamaria Baquero; BRUN, Marilia Maroneze; BARROS, Eder Marcos L.; MARTINS, Vanessa M.; ROSENBERG, Jhon P.; YATES, Patsy. Identification and characteristics of patients with palliative care needs in Brazilian primary care. BMC Palliat Care. v.51, n.15. p.1-10. 2016. Disponível em: < https://www.ncbi.nlm.nih.gov/pmc/articles/PMC4888621/pdf/12904_2016_Article_12 5.pdf $>$. Acesso em 17 ago 2017.

OLIVEIRA, SG.; SARTOR, SF.; MORAIS, ES.; MAYA, NF.; GERVINI, CMB.; MORALES CPM. Espiritualidade, religiosidade e terminalidade: temas possíveis nas visitas domiciliares realizadas a cuidadores familiares. Rev Enferm UFPI. v. 6 , n.1, p.69$73 . \quad$ Disponível em: < http://www.ojs.ufpi.br/index.php/reufpi/article/view/5800/pdf.>. Acesso em 16 ago 2017.

POTTER, Patricia; PERRY, Anne Griffin. Fundamentos de Enfermagem. $8^{a}$ ed. São Paulo: Elsevier; 2013.

SOUSA, Aurora Daniela Almeida de. Sintomas em cuidados paliativos: da avaliação ao controlo. Dissertação de Mestrado em Oncologia. Instituto de Ciências Biomédicas Abel Salazar. Universidade do Porto. Portugal, 151 p. 2012. Disponível em: <https:// repositorio-aberto.up.pt/bitstream/10216/65130/2/30465.pdf>. Acesso em 25 Nov 2018.

UNIC, Unidade de Cuidados Paliativos. Manual de cuidados paliativos em pacientes com câncer. Rio de Janeiro: UNATI/ UERJ- Univ. Aberta $3^{\text {a }}$ Idade. $1^{\text {a }}$ ed, 2009.

ZIMMERMANN, Camilla.; SWAMI, Nadia.; KRYZANOWSKA, Monika.; LEIGHL, Natasha.; RYDALL, Anne.; RODIN, Gary.; TANNOCK, Ian.; HANNON, Breffni. Perceptions of palliative care among patients with advanced cancer and their caregivers. CMAJ. V.188, n.10. p. 217-27. 2016. Disponível em: < http://www.cmaj.ca/content/188/10/E217.full.pdf+html>. Acesso em 17 Ago 2017.

Recebido em: 06/11/2017

Aceito em: 11/12/2018 